\title{
CLINICAL PROFILE OF PATIENTS WITH PARTIAL SEIZURE
}

\section{Dilli Ram Kafle}

\begin{abstract}
In partial seizure the epileptic discharge begins in one area of the brain and may or may not spread to other areas of the brain. When the discharge spreads to both the hemisphere it may lead to partial seizure with secondary generalization.It is a descreptive cross sectional study carried out at Tribhuvan University Teaching Hospital between April 2012 to April 2013. A total of 70 patients with partial seizure attended medical opd and admitted to medical ward. There were 40 males and 30 females. Age distribution of patient's presentation with partial seizure was as follows. Less then 20 years of age: 27 patients. 20-40:24 Patients. 41-60: 21 Patients. 60-80: 8 patients. 20 patients had simple partial seizure. 20 patients had complex partial seizure: 30 patients had partial seizure with secondary generalization. Age at onset of partial seizure: Less than 20 years:45 Patients. 21-40 years: 12 patients 41-60 years:20 patients. More than 60 years: 3 patients. Duration before starting treatment: Less than 6 months:33 Patients. 6 month - 1year: 6 patients. More than 1 year: 31 Patients.Status epilepticus: 3 patients.Drug history: carbamazepine, Phenytoin.
\end{abstract}

EEG : Normal in 36 patients.Abnormal in 34 patients.Neuroimaging: Tuberculoma: 6 patients, Calcifed granuloma:39 patients,Tuberous sclerosis:2 patients,mesial temporal sclerosis:3 Patients,Cerebral infarction:12 Patients.Normal :18 patients.Family history of seizure was present in 10 patients.

Key word: Seizure, Electroencephalography, Epilepsy.

\section{Introduction}

Epilepsy is one of the most common neurologic problems worldwide. In recent years, important advances have been made in the diagnosis and treatment of seizure disorders. ${ }^{1}$ However, our understanding of the cellular and molecular mechanisms by which epilepsy develops, or epileptogenesis, is still incomplete.

\section{Classification of Epilepsy}

The term "epilepsy" encompasses a number of different syndromes whose cardinal feature is a predisposition to recurrent unprovoked seizures. Although specific seizures can be classified according to their clinical features (e.g., complex partial seizures and generalized tonic-clonic seizures), ${ }^{2}$ epilepsy syndromes can also be classified according to the type of seizure, the presence or absence of neurologic or developmental abnormalities, and electroencephalographic (EEG) findings. ${ }^{3}$ For example, the syndrome of juvenile myoclonic epilepsy is characterized by the onset of myoclonic seizures, generalized tonic-clonic seizures, and less frequently absence seizures in adolescents who have normal intellectual function, with EEG findings of rapid, generalized spike-wave and polyspike-wave discharges. $^{4}$

Epilepsy syndromes fall into two broad categories: generalized and partial (or localization-related) syndromes. ${ }^{3,4} \quad$ In generalized epilepsies, the predominant type 
of seizures begins simultaneously in both cerebral hemispheres. Many forms of generalized epilepsy have a strong genetic component; in most, neurologic function is normal. In partial epilepsies, by contrast, seizures originate in one or more localized foci, although they can spread to involve the entire brain. Most partial epilepsies are believed to be the result of one or more central nervous system insults, but in many cases the nature of the insult is never identified.

\section{Mechanism of Partial epilepsy Mesial Temporal-Lobe Epilepsy}

We know much less about the mechanisms underlying partial-seizure disorders than we do about generalized epileptogenesis, even though partial seizures are the most common seizure disorder in adults, often stemming from focal lesions such as head trauma, strokes, and tumors. ${ }^{5}$ The most prevalent of these syndromes features complex partial seizures arising from the mesial temporal lobe., ${ }^{4,6}$ Recordings from intracranial depth electrodes have clearly demonstrated an ictal onset in mesial temporal structures such as the hippocampus, amygdala, and adjacent parahippocampal cortex; surgical resection of these areas in suitable patients usually abolishes the seizures. ${ }^{7}$ These seizures can begin with olfactory or gustatory hallucinations, an epigastric sensation, or psychic symptoms such as déjà vu or depersonalization. Once the seizures progress to a loss of awareness, the patients may stare blankly, speak unintelligibly, or exhibit lip smacking, picking at clothing, or other automatisms. $^{8}$

Although studies of mesial temporal-lobe epilepsy have yielded useful information, there are other plausible mechanisms of partial epileptogenesis that are not suggested by this syndrome. Some partial epilepsies, for example, are genetically determined.
Autosomal dominant nocturnal frontal-lobe epilepsy, in which single-gene mutations have been identified, has turned out to be a channelopathy that affects the neuronal nicotinic acetylcholine receptor, which serves as a ligand-gated sodium channel. ${ }^{9}$ Why mutations in this receptor, which is widely expressed throughout the brain, should cause partial seizures in the frontal lobe is just one of many mysteries. Some genetically determined partial epilepsies, such as benign epilepsy with centrotemporal spikes (also called benign rolandic epilepsy), are agelimited syndromes, suggesting the importance of developmental influences. ${ }^{4,10,11}$ For many patients with partial epilepsy, there may be an underlying genetic predisposition that becomes manifest only after a sufficient environmental insult. Obviously, understanding what molecular mechanisms are at work in patients with such a predisposition is of considerable clinical interest.

\section{Methodology}

It was a descriptive analytical crosssectional study carried out at Tribhuvan University Teaching Hospital. Clinical characteristics of all the patients attending medical OPD and patients admitted to medical ward with history of partial seizure were recorded between from April 2012 to April 2013.

\section{Result}

From April 2012 to April 2013 a total of 70 patients with partial seizure attended medical opd and admitted to medical ward.There were 40 males and 30 females.Age distribution of patient's presentation with partial seizure was as follows.Less then 20 years of age: 27 patients. 20-40:24 Patients. 41-60: 21 Patients. 60-80: 8 patients.Simple Partial seizure: 20.Complex Partial seizure: 20 
Partial seizure with secondary generalization:30.Age at onset of partial seizure: Less than 20 years:45 Patients. 21-40 years: 12 patients, $41-60$ years:20 patients and more than 60 years:3 patients. Duration before starting treatment:Less than 6 months:33 Patients: 6 month - 1year:6 patients and more than 1 year: 31 Patients. 3 patients had history of Status epilepticus. Most of the patients were taking carbamazepine, Phenytoin as monotherapy.

EEG : Normal in 36 patients.Abnormal in 34 patients.

Neuroimaging: Tuberculoma: 6 patients. Calcifed granuloma:39 patients.Tuberous sclerosis: 2 patients.

Mesial temporal sclerosis: 3 Patients. Cerebral infarction: 12 Patients. Normal :18 patients.

Family history of seizure: 10 patients.

\section{Discussion:}

The study highlights the current scenario of the pattern of seizure cases in a tertiary care .The fact that seizures affects commonly the productive age group and occurs due to secondary causes in over a third of cases are issues for concern.

A recent meta-analysis study showed that the age-specific prevalence rates were higher in the younger age group, with the onset of epileptic seizures reported mostly in the first three decades of the sample population's lives. [12] Mean age of onset of epilepsy was 14.8 years in contrast to our study (25 years). ${ }^{[13]}$ Family history of epilepsy in a study carried out in Sudan was present in $20 \%$ cases, which were higher than our observations of $10 \%$.

The common causes of partial seizure in patients attending to medical opd or admitted to medical ward in decreasing order was calcified granuloma:39 ,Cerebral infarction:12 Patients; Tuberculoma: 6 patients, Mesial temporal sclerosis:3 Patients and tuberous sclerosis:2
patients.Neuroimaging was normal in 18 patients.

EEG was abnormal in $48.6 \%$ of patients. This was comparable to a study carried out by Joseph et all in india in which EEG was abnormal in $47.1 \%$ of patients. ${ }^{14}$

Majority of patients were maintained on monotherapy of phenytoin or carbamazepine.

\section{References}

1. Schachter SC. Epilepsy. Neurol Clin 2001;19:57-78

2. Commission on Classification and Terminology of the International League against Epilepsy. Proposal for revised clinical and electroencephalographic classification of epileptic seizures. Epilepsia 1981; 22: 489-501

3. Commission on Classification and Terminology of the International League against Epilepsy. Proposal for revised classification of epilepsies and epileptic syndromes. Epilepsia 1989;30:389-399

4. Benbadis SR. Epileptic seizures and syndromes. Neurol Clin 2001;19:251-270

5. Annegers JF. The epidemiology of epilepsy. In: Wyllie E, ed. The treatment of epilepsy: principles and practice. 3rd ed. Philadelphia: Lippincott Williams \& Wilkins, 2001:131-8.

6. Engel J Jr. Mesial temporal lobe epilepsy: what have we learned? Neuroscientist 2001;7:340-352

7. Kim R, Spencer D. Surgery for mesial temporal sclerosis. In: Lüders HO, Comair YG. Epilepsy surgery. 2nd ed. Philadelphia: Lippincott Williams \& Wilkins, 2001:643-52.

8. French JA, Williamson PD, Thadani VM, et al. Characteristics of medial temporal lobe epilepsy. I. Results of history and physical examination. Ann Neurol 1993;34:774-780

9. Steinlein OK, Mulley JC, Propping P, et al. A missense mutation in the neuronal nicotinic acetylcholine receptor alpha 4 subunit is associated with autosomal dominant nocturnal frontal lobeepilepsy.NatGenet1995;11:201-203

10. Sanchez RM, Jensen FE. Maturational aspects of epilepsy mechanisms and consequences for the immature brain. Epilepsia 2001;42:577-585

11. Ottman R. Progress in the genetics of the partial epilepsies. Epilepsia 2001;42:Suppl 5:24-30

12. Sridharan R, Murthy BN. Prevalence and pattern of epilepsy in India. Epilepsia 1999;40:631-6 
13. Thomas SV, Sarma PS, Alexander M, Pandit L, Shekhar L, Trivedi C, et al. Epilepsy care in six Indian cities: A multicenter study on management and service. J Neurol Sci 2001;188:73-7.
14. Nitin Joseph, Ganesh S Kumar, Maria Nelliyanil. Pattern of seizure cases in tertiary care hospitals in Karnataka state of India.Ann of Indian Academy of Neurol 2013; 16:347-35

Corresponding author: Dr. Dilli Ram Kafle, Institute of Medicine,Maharajgunj Kathmandu, Nepal; E-mail:kafledilli@yahoo.com 